\title{
La política cambiaria de América Latina en el contexto de baja cooperación mo- netaria internacional ${ }^{1}$
}

\author{
Latin American Exchange Rate Policy in the Context \\ of Low International Monetary Cooperation
}

\author{
Susana Nudelsman ${ }^{2}$
}

Resumen: La cooperación monetaria en el contexto del sistema monetario internacional posterior a los Acuerdos de Bretton Woods es baja. Aunque el régimen de tipos de cambio flotantes prometía ajustes menos traumáticos que su predecesor, varios factores concurrieron para la obtención de resultados de signo contrario. La mayor flexibilidad de las políticas cambiarias de América Latina partir de los 2000, así como la acumulación de reservas externas, contribuyeron a sortear la crisis financiera global de manera más eficiente que los shocks exógenos del pasado. A nivel global, la acumulación de reservas alimenta el problema de los desequilibrios globales mientras que la existencia de una multiplicidad de acuerdos cambiarios torna el sistema monetario internacional en un universo complejo.

Palabras claves: Tipos de cambio, Acumulación de reservas, Arquitectura financiera internacional, Cooperación monetaria internacional

\begin{abstract}
Monetary cooperation in the context of the international monetary system following the Bretton Woods Agreements is low. Although the floating exchange rate regime promised less traumatic adjustments than its predecessor, several factors concurred obtaining opposite sign results. The greater flexibility of exchange rate policies in Latin America since the 2000s, as well as the accumulation of external reserves, helped to overcome the global financial crisis more efficiently than in past exogenous shocks. At the global level, the accumulation of reserves feeds the problem of global imbalance, while the existence of a multiplicity of exchange rate agreements makes the international monetary system a complex universe.
\end{abstract}

Keywords: Exchange Rates, Reserves Accumulation, International Financial Architecture, International Monetary Cooperation

\footnotetext{
Doi: https://doi.org/10.24215/23142766e069

${ }^{1}$ Recibido: 05/08/2018. Aceptado: 15/02/2019

${ }^{2}$ Doctora en Economía, UBA. Estudios de Economía Internacional, Graduate Institute, Ginebra. Trayectoria de docencia e investigación en UBA y en universidades de EEUU. Ex-becaria del CONICET, la Comisión Fulbright, Swiss Agency for Development and Cooperation y LASA. Miembro del CARI y del T20 Argentina 2018. Evaluadora de revistas de Economía.
} 


\section{Introducción}

En el contexto de un aumento notable de la movilidad del capital, el sistema monetario internacional surgido de los Acuerdos de Bretton Woods era insostenible. Los controles de capital que acompañaron a los tipos de cambio fijos pero ajustables permitían a los gobiernos alterar las condiciones financieras internas a fin de alcanzar los otros objetivos sin poner en peligro inmediatamente la estabilidad del tipo de cambio. Los controles también contribuían a la reconstrucción del comercio internacional; sin embargo, la conjunción del libre comercio y el control de los movimientos financieros internacionales mostraron una dinámica inestable. Con la liberalización de los mercados financieros internacionales, disminuyó la viabilidad de controlar los movimientos de capital, lo que ejerció presión sobre dicho sistema de tipos de cambio (Eichengreen, 2008:238-239).

A finales de la década de los sesenta, la frecuencia de las crisis monetarias llevó a los economistas a defender una mayor flexibilidad de los tipos de cambio. Se sostenía que el establecimiento de los tipos de cambio flotantes no solo aseguraría automáticamente la flexibilidad del tipo de cambio, sino que también produciría beneficios para la economía global. Los países podrían utilizar la política monetaria para alcanzar el equilibrio interno y externo al tiempo que las asimetrías inherentes al sistema de Bretton Woods desaparecerían (Krugman, 2012:543).

Sin embargo, el sistema monetario internacional actual tiene una serie de problemas que están interrelacionados entre sí. En efecto, este exhibe una alta volatilidad y un amplio y persistente desalineamiento de los tipos de cambio, una falla para promover mayor coordinación de políticas económicas entre los países más avanzados y una incapacidad para prevenir crisis financieras internacionales o para lidiar con ellas una vez que emergen (Salvatore, 2011:761). En particular, el problema de los desequilibrios globales no es un fenómeno nuevo, aunque su naturaleza ha cambiado desde los años de la "escasez de dólares" hasta los años actuales de "exceso de dólares". Los problemas generados por tales desequilibrios globales han sido fuente persistente de preocupación a lo largo del tiempo y han dado lugar a intensos debates acerca de la necesidad de mecanismos de ajuste apropiados, con primordial énfasis sobre el tipo de cambio (Ocampo, 2016:1).

Por su parte, la integración financiera global ha permitido la movilización de vastos montos de recursos y contribuido a la incorporación de nuevas economías emergentes en la economía global. No obstante, también ha contribuido a generar graves crisis financieras tanto en países en desarrollo como en países desarrollados. Ello fue particularmente comple-jo para los primeros. Las economías de América Latina, así como las economías en desarrollo más generalmente, han acudido a la práctica de acumular reservas externas a fin de protegerse ante adversidades externas. Asimismo, las economías de la región han ido abandonando las anclas cambiarias para abrazar regímenes cambiarios más flexibles desde comienzos del nuevo milenio. A diferencia de lo acontecido en el pasado, estas economías hoy cuentan con más herramientas de política económica para enfrentar momentos críticos (de la Torre, Levy Yeyati y Pienknagura, 2013:44).

No obstante, la configuración monetaria global pone en evidencia que la práctica de acumulación de reservas y la proliferación de arreglos cambiarios generan tensiones significativas. Aunque dicha práctica es totalmente racional para los países individualmente, esto no sucede a nivel global en tanto esta se corresponde con los desequilibrios globales, uno 
de los aspectos distintivos de la reciente crisis global. Por su parte, la proliferación de arreglos cambiarios ha convertido el sistema monetario internacional en una suerte de "no-sistema" (Williamson, 1977:73; Ocampo 2017:100).

El presente artículo considera oportuno reflexionar sobre ciertos aspectos cruciales de la arquitectura financiera internacional. Ellos se refieren a la caracterización de la cooperación internacional, los arreglos cambiarios post Bretton Woods, las asimetrías del sistema monetario internacional, las motivaciones de la acumulación de reservas, la centralidad de los tipos de cambio, las políticas cambiarias de América Latina y un panorama global al respecto. Finalmente, se presentan las conclusiones.

\section{La cooperación monetaria internacional}

El análisis de las relaciones monetarias subraya el papel de la cooperación, ya sea a nivel de la operatoria de las finanzas globales como de su gobernabilidad. La cooperación entendida como una estructura de responsabilidades y toma de decisiones compartidas puede materializarse de diversas formas e incluir desde redes de negociación informales hasta procedimientos más formalmente establecidos, lo que permite corregir las fallas del mercado internalizando las sus externalidades. En la medida en que cada gobierno puede ejercer cierto grado de control sobre los otros, es posible moverse hacia una política colectiva cercana a lo que puede entenderse como un óptimo de Pareto (Cohen, 2002:435-437). En particular, los beneficios mutuos de la cooperación surgen de la posibilidad de comunicación y coordinación entre los participantes del sistema (Hamada, 1985:20).

En principio, los diversos gobiernos pueden potencialmente beneficiarse si actúan de manera colectiva; sin embargo, en la práctica, la cooperación monetaria internacional es limitada. La solución a este dilema consiste en encontrar algún tipo de acuerdo sobre las reglas que tengan posibilidad de ponerse en vigencia (Martin y Simmons, 1998:737). Se ha subrayado que debe distinguirse entre los "dilemas de aversiones comunes" y los "dilemas de intereses comunes" (Stein, 1990:32-38). Afortunadamente, en muchas dimensiones de las finanzas internacionales, los primeros prevalecen por sobre los segundos. De esta manera, en algunos aspectos, tales como la supervisión y la regulación de los mercados financieros, la cooperación internacional ha mostrado importantes avances (Frieden, 2017:532-534).

En otras dimensiones de la arquitectura financiera internacional, los patrones de cooperación son más complejos. Por un lado, dicha política en tanto mecanismo de auto-protección por parte de los países en desarrollo para resguardarse frente a shocks externos es sumamente ventajosa; por otro lado, también alimenta los desequilibrios globales. Asimismo, la multiplicidad de acuerdos cambiarios tras el fin del sistema monetario internacional instituido en Bretton Woods ha dado por resultado un sistema falto de reglas bien definidas en materia cambiaria (Williamson, 1977:74). Aunque imperfecto, este "no-sistema" ha mostrado una considerable resiliencia y ha experimentado importantes avances; sin embargo, aún exhibe deficiencias en las cuales subyace un sistema monetario internacional asimétrico Ocampo (2016:31). Una mayor cooperación internacional que mitigue la generación de desequilibrios globales y la volatilidad de los tipos de cambio es deseable. 


\section{La era post Bretton Woods}

Tras la caída de los Acuerdos de Bretton Woods, en 1973 el sistema monetario internacional de tipos de cambio flotantes inauguró una etapa de grandes transformacio-nes. El nuevo régimen apuntaba a que la política de los bancos centrales y los gobiernos tendiera más a la flotación de los tipos de cambio que a su estabilidad. Los hacedores de política estarían dispuestos a hacer pleno uso del instrumento de política monetaria a su disposición en el marco del nuevo esquema monetario.

En un inicio, el mantenimiento de los controles de capital permitió compatibilizar el objetivo de la estabilidad cambiaria con otros objetivos de política, tales como los programas de reconstrucción europea en el corto plazo y el pleno empleo en el largo plazo. Sin embargo, con el transcurso del tiempo, la efectividad de dichos controles fue cayendo. Liberados de las limitaciones a la movilidad del capital, resultaba problemático conciliar la creciente recuperación de los mercados financieros internacionales con la operatoria de los tipos de cambio fijos pero ajustables. En un mundo de alta movilidad del capital, la defensa de la paridad requería una fuerte intervención en los mercados cambiarios que no todos los países estaban dispuestos a apoyar sin antes conocer la voluntad o capacidad de los gobiernos por alejar la fuente del desequilibrio de pagos (Eichengreen, 2008:134-136).

No obstante, el pasaje desde el sistema Bretton Woods al de los tipos de cambio flotantes fue lento. En este proceso, algunos países adhirieron a los tipos de cambio flotantes al tiempo que otros tendieron a la estabilidad. El cambio fue mayor para los países más avanzados que abogaron por la flotación, aunque algunos dentro de este conjunto prefirieron fijar sus monedas entre ellos y flotar frente a terceras monedas, a fin de promover el comercio en el proceso de integración. En los países en desarrollo, el pasaje a la flexibilidad cambiaria fue menos abrupto, pero la fijación cambiaria ha ido perdiendo preponderancia (Eichengreen, 2008: 228).

En la actualidad, la flotación cambiaria no es aplicada a escala global de manera uniforme. Preferentemente, la economía internacional se caracteriza por la coexistencia de diversos arreglos cambiarios. Tras el fin de los Acuerdos de Bretton Woods, ha habido un progresivo, aunque importante movimiento hacia regímenes cambiarios más flexibles. Sin embargo, los regímenes intermedios que combinan la flotación con intervenciones en los mercados cambiarios representan una proporción significativa del total (Ilzetzki, Reinhart y Rogoff, 2019:599).

Aunque el cambio hacia un sistema de tipos de cambio flotantes prometía un mundo con ajustes menos complejos, la falta de consenso en la construcción de un sistema monetario internacional sobre la base de reglas coherentes dio por resultado una pluralidad de acuerdos que hacen del mundo post Bretton Woods una suerte de "no-sistema" (WiIliamson, 1977:73; Ocampo, 2017:100).

Al finalizar el siglo $\mathrm{XX}$, el proceso de integración financiera global exhibe un rápido movimiento de activos altamente líquidos como característica distintiva. Por cierto, tal grado de movilidad del capital no se había registrado desde comienzos de dicho siglo y fines del precedente. A su vez, las manías, pánicos y crisis han recrudecido en las últimas décadas, lo que ha afectado tanto a países en desarrollo como desarrollados. 


\section{Asimetrías del sistema monetario internacional}

Entre los varios argumentos a favor del establecimiento de un sistema de tipos de cambio flotantes, se sostenía que estos eliminarían las asimetrías que caracterizaban el sistema de Bretton Woods derivadas del papel central del dólar en el sistema monetario internacional. Una se refería al hecho de que cualquier país podía devaluar su moneda frente al dólar en condiciones de "desequilibrio fundamental" mientras que las reglas del sistema impedían a Estados Unidos devaluar respecto en relación con las demás monedas extranjeras. La segunda se refería al hecho de que, al fijar los bancos centrales sus monedas respecto al dólar y asimismo acumular dólares como reservas externas, la Reserva Federal desempeñaba el papel primordial en la determinación de la oferta monetaria mundial (Krugman, Obstfeld y Melitz, 2012:544-545).

Sin embargo, el sistema monetario internacional aún exhibe asimetrías. Un primer problema surge del ajuste asimétrico del balance de pagos entre países deficitarios y superavitarios. En los debates anteriores al establecimiento de los Acuerdos de Bretton Woods, Keynes ya había destacado que todos los sistemas monetarios internacionales ponen la carga del ajuste macroeconómico sobre los países deficitarios. En ausencia de niveles apropiados de financiación externa o de reservas externas, los países deficitarios se ven forzados a ajustar, mientras que los superavitarios experimentan menor o incluso ninguna presión. Tal ajuste asimétrico genera, a su vez, un efecto contractivo global particularmente fuerte durante las crisis globales. Así, por ejemplo, en ocasión de la crisis global financiera de 20082009, los países de la periferia europea han llevado a cabo un notable ajuste y pasado de cuentas corriente deficitarias a superavitarias un lustro después. En cambio, los países superavitarios como Alemania y Holanda han mantenido su statu quo. Además, la situación difiere cuando se toma en cuenta el caso de los países deficitarios emisores de moneda de reserva - tal como el caso de Estados Unidos - versus los que no lo son (Ocampo, 2016:2122; Ocampo, 2017:47-49).

Un segundo problema surge del llamado "dilema de Triffin", es decir, la inestabilidad asociada al uso de una moneda nacional como moneda internacional. En el marco del patrón cambio oro, Triffin (1968:195-202) señalaba la amenaza de que el agotamiento de las reservas de oro de Estados Unidos forzaría a este país a adoptar políticas contractivas, lo que no ocurre actualmente en el régimen de dólar fiduciario. En efecto, este régimen no ha eliminado el dilema, sino que ha cambiado sus características. En la actualidad la deficiencia consiste en que la economía mundial se halla sujeta a la política monetaria completamente autónoma del país emisor de la moneda de reserva dominante. Liberado de la restricción de la convertibilidad de dólares a oro, Estados Unidos puede incurrir en déficits de manera persistente como ha venido ocurriendo desde los años ochenta. A diferencia de lo notado por Keynes, el sistema de dólar fiduciario puede también generar efectos expansivos más que contractivos particularmente durante los períodos de auge del ciclo económico. Puesto que este sistema ha generado gran volatilidad en la cuenta corriente y en el tipo de cambio de Estados Unidos, el dólar ha perdido estabilidad al tiempo que la generación de liquidez internacional es más errática actualmente que en el sistema de Bretton Woods (Ocampo, 2017:49-53).

Otro problema proviene del "sesgo hacia la desigualdad" (Ocampo, 2017: 53-59). Las reservas en moneda extranjera de los países en desarrollo son invertidas en activos emitidos por los países ricos, lo que implica prestar a dichos países a tasas de interés bajas. Tras la crisis 
asiática, los países en desarrollo han estado acumulando vastos montos de reservas a fin de enfrentar crisis tanto de balanza de pagos como financieras originadas por reversiones súbitas de los flujos de capital. La acumulación de reservas o "auto-seguro" como política macroeconómica contra-cíclica ante los shocks de los flujos de capital pro-cíclicos - como de los patrones pro-cíclicos de los precios de los productos básicos y, en menor medida, del volumen del comercio internacional - tiene lógica para los países individuales y ciertamente ha ayudado a muchos de ellos a resistir las turbulencias financieras externas recientes de mejor manera que en el pasado. No obstante, según lo advierte el Informe del Comité Zedillo de las Naciones Unidas (2001), dicha política también involucra una falacia. Por un lado, la pro-ciclicalidad de los flujos de capital y la ausencia de un mecanismo adecuado para asegurarse frente a las interrupciones bruscas de financiamiento externo justifican la lógica de la estrategia de acumulación de reservas puesta en práctica por la mayoría de países en desarrollo. Por otro lado, esta estrategia implica una redistribución de ingresos desde estos países hacia los más avanzados por lo que se considera que los primeros han recibido ayuda inversa.

Al mismo tiempo, la acumulación de reservas empeora los desequilibrios globales pudiendo generar problemas en la estabilidad financiera global. Sin embargo, la crisis global ha supuesto un ajuste parcial de dichos desequilibrios y de algunas de sus causas subyacentes. Cabe notar que, a pesar de la aparente insostenibilidad de tales desequilibrios en el largo plazo, estos perduran en el tiempo. Más aún, las elevadas tenencias de reservas en dólares por parte de los bancos centrales de los países en desarrollo generan incentivos a la persistencia de la política de acumulación en tanto una apreciación de las monedas nacionales generaría una pérdida de valor de los activos en dólares (Alberola y Serena, 2010:33-34).

Entre las imperfecciones de los mercados financieros internacionales, la literatura también ha enfatizado el fenómeno del "pecado original" (Eichengreen, Hausmann y Panizza, 2002:1-6). Con excepción de los países emisores de las monedas principales, la mayoría de los países no puede tomar préstamos en sus propias monedas, lo que generalmente causa que experimenten dificultades en términos de inestabilidad financiera y macroeconómica. La acumulación de deuda neta denominada en moneda extranjera por parte de los países en desarrollo se traduce en balances con descalces cambiarios agregados. Estos países exhiben mayor volatilidad en el producto y en los flujos de capital, calificaciones crediticias más bajas y políticas monetarias más rígidas y deben afrontar una prima de riesgo adicional cuando toman prestado. Un shock al tipo de cambio puede mover adversamente los precios de los activos mientras que cualquier intento por minimizar sus riesgos limitando el uso de financiamiento afectará su proceso de crecimiento. El "pecado original" no puede atribuirse totalmente a la debilidad de las políticas e instituciones nacionales. Los factores que limitan los incentivos a la diversificación de divisas por parte de los inversores globales - costos de transacción en un mundo de países heterogéneos o externalidades de redtambién importan. El concepto de que la integración financiera internacional canalizaría el capital hacia los países en desarrollo y, por lo tanto, promovería el crecimiento ha encontrado muchos obstáculos en los hechos. Se trata no solo de un problema de las políticas nacionales sino también de la operatoria de la arquitectura financiera internacional. La mayor frecuencia e intensidad de las crisis financieras sugeriría que la inestabilidad tiene, en gran parte, importantes raíces sistémicas.

El tema del "privilegio exorbitante" (Eichengreen, 2011:1-8) también está en juego. Estados Unidos, centro del sistema monetario internacional, percibe señoreaje por la emisión de dólares mientras que los miles de millones de moneda estadounidense que circulan 
fuera de él proporcionan igual monto en la misma moneda en bienes y servicios reales. Por cierto, cuesta solo unos pocos centavos la producción de un billete de cien dólares por la oficina encargada en dicho país mientras que otros países tienen que pagar hasta cien dólares de bienes y servicios reales para obtener uno. Originalmente acuñado por Giscard d'Estaing en los años sesenta, el concepto de "privilegio exorbitante" enunciaba que el centro del sistema era fuertemente asimétrico en términos financieros. Aún hoy las críticas hacen foco en el hecho de que cualquier país que vive más allá de sus posibilidades debe devaluar su moneda, emitir más moneda para pagar sus cuentas, hacer frente a la inflación subsiguiente y reducir sus gastos. De este modo, el privilegio en cuestión permitió a Estados Unidos financiar sus déficits externos durante las últimas décadas.

\section{La acumulación de reservas externas}

La transición del patrón dólar fijo al de dólar flotante inauguró una tendencia creciente en la adopción de arreglos cambiarios más flexibles. Al mismo tiempo, la demanda de reservas externas fue aumentando a través de los años. La necesidad de reservas es obvia en los regímenes de tipos de cambio fijos. Por cierto, los modelos a la Krugman (1979:323-324) enfatizan las limitaciones de los gobiernos que utilizan las reservas en la provisión de un ancla nominal. No obstante, la evidencia pone de manifiesto que la acumulación de reservas es una práctica aplicable a cualquier régimen de política cambiaria extendida tanto a países en desarrollo como desarrollados. La explicación de esta paradoja debe tomar en cuenta que el tránsito hacia la flotación, tras el colapso de Bretton Woods, tuvo lugar en el marco de una colosal liberalización financiera. Circunstancias tales como la mayor volatilidad, frenazos súbitos de capital, contagio y proclividad a las crisis justifican nuevos usos para las reservas internacionales (Redrado, 2006:19).

En relación con la acumulación de reservas, un primer punto de vista destaca los motivos precautorios de tipo tradicional. En el marco de la teoría cuantitativa del dinero, hacia los años treinta se explicaba la acumulación de reservas como proporción a la producción y el comercio (Olivera, 1969:246). Por su parte, Triffin (1947:72-73) propuso emplear el cociente entre reservas e importaciones como un indicador apropiado a fin de poder financiar los déficits previsibles de los pagos internacionales mientras se espera una mejora del balance de pagos en caso de un desequilibrio temporario o el efecto del ajuste de cualquier política correctiva cuando el déficit es de naturaleza persistente. En una etapa posterior, el enfoque monetarista del balance de pagos explicó la demanda de reservas en función del exceso de demanda de dinero (Johnson, 1958:157).

Un segundo punto de vista surge tras las crisis asiáticas, con los problemas financieros derivados de la apertura de la cuenta capital. Por cierto, la fuente de riesgo externo principal mutó desde la cuenta corriente a la cuenta de capital. La interrupción súbita del financiamiento, el contagio y la volatilidad son fuentes de alta incertidumbre en el nuevo escenario global. El motivo precautorio por el lado financiero ejerce su influencia como reaseguro para economías que ante shocks de la cuenta de capital encuentran obstáculos en el acceso al mercado de crédito (Aizenman y Lee, 2005:3). A fin de reducir la probabilidad de crisis, ampliar el margen de maniobra o llevar a cabo un ajuste menos traumático, la acumulación de reservas parece ser un mecanismo más apropiado que la asistencia de los organismos internacionales (Feldstein, 1999:2). A su vez, cuando los datos fundamentales se encuentran en 
una zona intermedia según lo explicado por los modelos de segunda generación de crisis, resulta oportuno fortalecer los niveles de reservas (Li y Rajan, 2005:11-12).

Un tercer punto de vista analiza la acumulación de reservas como subproducto del régimen cambiario. Este carácter "derivado" de la demanda de reservas se explica en función del motivo mercantilista donde los países buscan evitar la apreciación de la moneda a fin de proteger su industria de los competidores internacionales. La devaluación de la moneda, en cambio, estimula el crecimiento económico -particularmente en los países en desarrollo, con el este de Asia como el caso más emblemático-, siendo precisamente el sector transable -especialmente la industria- el canal operativo por el cual se pone en práctica este proceso (Rodrik, 2008:404-409).

Resulta difícil determinar si la demanda de reservas se debe a una única motivación. Es bastante probable que coexistan diferentes motivaciones. Sin embargo, existe consenso en que, en los países en desarrollo, el auto-seguro juega un papel decisivo. Desde la crisis asiática, estos países han llevado a cabo una acumulación sistemática de reservas externas.

\section{Centralidad de las políticas cambiarias}

Tras la liberalización de los mercados financieros, la rápida expansión de los flujos de capital ha contribuido al crecimiento económico de muchos países en desarrollo. Sin embargo, la inestabilidad de dichos flujos también ha contribuido a la generación de serios episodios de crisis, en los cuales los tipos de cambio constituyeron un aspecto central de dicha dinámica (Krugman, 2000:1).

La centralidad de los tipos de cambio se origina en tres factores diferentes: sus efectos en el comercio internacional, su papel en la corrección de los desequilibrios de pagos globales y su papel potencial sobre la estabilidad macroeconómica de los países individuales. Respecto al primer factor, no existe un mecanismo vinculante entre el comercio mundial y las reglas cambiarias. Dada la significatividad macroeconómica de los tipos de cambio, se sugiere incluir este asunto en el mecanismo de solución de controversias de la Organización Mundial de Comercio. Este mecanismo no ha sido perfecto, pero la ausencia de instancias alternativas amerita tanto su uso como su mejora (Matoo y Subramanian, 2008:9).

En relación con el segundo factor, el sistema cambiario global actual ha fallado en cumplir dos objetivos establecidos en el Convenio Constitutivo del Fondo Monetario Internacional: la reducción del grado de desequilibrio de los balances de pagos internacionales y la promoción de la estabilidad cambiaria. Al respecto, un tema de debate consiste en dilucidar el papel de las variaciones cambiarias que contribuyen o no a corregir los desequilibrios globales. Algunos autores no encuentran una relación robusta entre los tipos de cambio flexibles y los ajustes de cuenta corriente (Chinn y Wei, 2008:23) mientras que otros, en cambio, han encontrado el resultado opuesto (Ghosh, Qureshi y Tsangarides, 2014:24).

Un tema de significación aún mayor es que, en el mundo actual, los tipos de cambio no siempre responden a los datos fundamentales de la economía sino a los flujos de capital y a los ciclos de expansión y contracción asociados a ellos. La evidencia sugiere que estos ciclos no están exentos de la posibilidad de equilibrios múltiples y profecías auto-cumplidas en los modelos de crisis. Por cierto, los tipos de cambio pueden imprevisiblemente desconectarse de su valor fundamental e involucrarse en la dinámica de burbujas y shocks (Williamson, 2008:11). 
Respecto al tercer factor, los tipos de cambio han sido un determinante crucial en la explicación de los numerosos eventos de crisis de los últimos tiempos. Desde la manía de los tulipanes, las crisis cambiarias han sido recurrentes en la historia económica moderna. Aunque jugaron un papel en el período comprendido entre las dos guerras, en la era posterior a los Acuerdos de Bretton Woods y en la crisis de la deuda latinoamericana, su impacto ha sido aún mayor desde los años noventa. La mayor integración de los mercados de capital en el marco de una ola creciente de innovaciones financieras en tiempos recientes ha agregado nuevos elementos de análisis.

Siguiendo a la literatura tradicional, los tipos de cambio fijos tienen la facultad de proveer mayor estabilidad de precios por la vía de la provisión de un ancla creíble para la política monetaria mientras que lo opuesto ocurre con los tipos de cambio flotantes. Los primeros entrañan la renuncia de la autonomía monetaria al tiempo que los segundos la hacen posible, lo que es sumamente útil para hacer frente a shocks externos e internos. Además, los tipos de cambio fijos contribuyen a minimizar el riesgo cambiario de los flujos de comercio globales, facilitar los flujos de capital globales, evitar las depreciaciones competitivas y eludir las burbujas especulativas. Por su parte, los tipos de cambio flotantes posibilitan un ajuste automático de los shocks comerciales, detentar el señoreaje y el papel de prestamista de última instancia por parte del banco central y prevenir los ataques especulativos. Tras la crisis asiática y las crisis posteriores en las economías emergentes, la literatura se focalizó en los mercados financieros y la estabilización de la especulación. La defensa de los tipos de cambio fijos se basó en la gran desventaja de la flotación consistente en la tendencia a la volatilidad no siempre causada por los fundamentos económicos. Por su parte, la defensa de los tipos de cambio flotantes se basó en la gran desventaja de la fijación consistente en la tendencia de los prestatarios a exponerse en moneda extranjera sin cobertura eficaz, lo que derivó en ataques especulativos y equilibrios múltiples. Sin embargo, la evidencia ha demostrado que ninguno de estos regímenes fue capaz de impedir ni la excesiva volatilidad ni la sobrevaluación cambiaria (Frankel, 2012:769-778).

Las soluciones extremas no son la panacea. Constituyen una solución extrema sujeta a dos demandas en conflicto - la estabilidad de precios nominales versus la flexibilidad de precios relativos. En cambio, la elección de los regímenes intermedios es el resultado del objetivo de conciliar estas opciones extremas (French Davis, 2003:20; Ocampo, 2002:11-12). Algunos acuerdos intermedios, especialmente aquellos con poca flexibilidad, involucran riesgos de crisis mientras que otros, como los de flotación administrada, reducen tales riesgos al tiempo que proporcionan autonomía monetaria. No obstante, el concepto de flotación administrada abarca un espectro amplio de acuerdos cambiarias lo que impide distinguir entre flotaciones seguras y regímenes intermedios riesgosos (Ghosh, Ostry y Qureshi, 2014:21).

La experiencia muestra que la elección pragmática de los acuerdos intermedios es sumamente útil para los países emergentes. Ello incluye intervenciones cambiarias con el fin de corregir la desalineación cambiaria, el manejo contra-cíclico de las reservas en moneda extranjera y, en algunos casos, el uso de regulaciones de la cuenta de capital (Ocampo, 2017:105-106). 


\section{Los tipos de cambio en América Latina}

Con el objetivo de abatir la inflación y la hiperinflación, los países de América Latina pusieron en marcha numerosas experiencias de regímenes de tipos de cambio. En los setenta, se aplicaron políticas de estabilización ortodoxas sobre la premisa de la reducción del gasto público. Los países del Cono Sur implementaron políticas basadas en el enfoque monetarista del balance de pagos para una economía cerrada. Siguiendo la apertura de estas economías, este enfoque fue girando a políticas de estabilización focalizadas en los tipos de cambio (Ramos, 1985:87).

En los ochenta, las políticas heterodoxas combinaron una política fiscal restrictiva con la fijación del tipo de cambio y políticas de ingresos para controlar precios y salarios. No obstante, la memoria del régimen de alta inflación estaba fuertemente arraigada (Frenkel, 1989:9-17). A pesar del éxito inicial de estos programas por abatir la inflación, la persistencia de la indexación, la puja distributiva y la dificultad en instrumentar disciplinas fiscal y monetaria junto al racionamiento del crédito externo en un escenario de elevada deuda externa frustraron los objetivos antiinflacionarios (Machinea y Fanelli, 1988:183-186).

En los noventa, varios países de la región, así como otros países en desarrollo, aplicaron regímenes de tipos de cambio fijos y semifijos. Sin embargo, los diferentes episodios de crisis financieras pusieron en evidencia los límites de los regímenes intermedios en el contexto de una rápida liberalización de la cuenta de capital y de una inadecuada regulación de los sistemas financieros nacionales. Las críticas a ambos tipos de regímenes eran diversas. Unos sostenían que, si estas economías no hubieran fijado sus monedas al dólar, habríaa sido posible evitar los ataques especulativos que derivaron en crisis financieras (Obstfeld y Rogoff, 1995:1-2). Otros argumentaban que, si los países hubieran adoptado regímenes cambiarios super-fijos, los eventos habrían evolucionado mejor (Hausmann y otros, 1999:3). Otra visión que combinaba ambas críticas aseveraba que los regímenes estables solo se logran en los extremos lo que ha dado en llamarse solución bipolar (Fischer, 2001:9-10).

En el marco de este debate, hacia fines de los noventa circulaban dos enfoques. Por un lado, el enfoque bipolar combinado con el miedo a flotar (Calvo y Reinhart, 1999:404405) condujeron a la idea de que los tipos de cambio fijos son los únicos viables para economías con alto grado de dolarización y sustitución de la moneda. A la sazón, golpeado por una situación crítica, Ecuador instrumentó en los inicios de los 2000 la dolarización de su economía. Por el otro lado, el fracaso con el plan de convertibilidad en Argentina mostró las restricciones de dicho régimen. Su sola implementación no garantizaba la obtención de la disciplina fiscal y monetaria ni el freno al proceso de dolarización (Frenkel y Rapetti, 2010:31-33). Aun cuando la economía había acumulado fragilidades de diversa índole, la fuerte apreciación de la moneda y el vertiginoso deterioro de la posición neta en divisas fueron factores decisivos del colapso de dicho régimen (Perry y Servén, 2003:1-3). En el marco de un proceso de desdolarización junto a una mayor disciplina monetaria que contribuyó a la lucha anti-inflacionaria y a una reducción de los coeficientes de passtrough, la flotación cambiaria pasó a ser la opción preferida para economías en proceso de integración financiera (Levy Yeyati, 2011:15).

En general, desde comienzos de los 2000, las economías de América Latina se han movido hacia regímenes cambiarios más flexibles y varios de ellos anunciaron la implementación de flotación con metas de inflación. La mayor flexibilidad cambiaria ha sido un importante avance en la región. A diferencia de lo ocurrido en episodios anteriores de crisis, en 
esta oportunidad los efectos contagio de la crisis financiera global en la región no desembocaron en crisis cambiarias. Por cierto, la mayoría de las economías latinoamericanas utilizó la devaluación del tipo de cambio como herramienta contracíclica para mitigar el impacto del shock externo. La mejora en el "sistema inmunológico macrofinanciero" posibilitó a varias economías de la región - particularmente a aquellas con regímenes cambiarios flexibles y políticas monetarias basadas en metas de inflación - acudir a las depreciaciones de las monedas nacionales a fin de absorber los shocks externos y alentar el nivel de actividad local (de la Torre, Levy Yeyati y Pienknagura, 2013:8). Al mismo tiempo, puesto que la política monetaria no se encontraba subordinada al comportamiento del tipo de cambio, la mayoría de los bancos centrales pudieron adoptar políticas monetarias contracíclicas reduciendo considerablemente la tasa de interés. Las políticas expansivas proveyeron la liquidez requerida por el sistema financiero interno, lo que permitió amortiguar el impacto negativo del shock sobre el crédito interno (Rojas-Suárez, 2010:8-9).

Sin embargo, no todos los países de la región han sido partícipes de la mejora macrofinanciera que les permite utilizar esta estrategia devaluatoria. Además, en algunos países las carteras de los agentes locales muestran una tendencia a la desnacionalización ante depreciaciones bruscas de los tipos de cambio. En tal caso, los efectos expansivos de una depreciación del tipo de cambio sobre la actividad y el empleo tienden a ser contrarrestados por una disminución del crédito y liquidez internos resultado de la caída de los depósitos en el sistema financiero local (Katz, 2009:132).

Cabe notar que la mayor flexibilidad de los tipos de cambio ha sido acompañada por intervenciones de los bancos centrales en los mercados cambiarios, ya sea para acumular reservas como autoseguro o para limitar el exceso de volatilidad cambiaria. En efecto, aunque la adopción de la flotación con metas de inflación se ha popularizado en América Latina, la intervención en los mercados cambiarios no estuvo ausente. Ninguna de las economías de la región ha instrumentado dicho arreglo monetario según su concepción primigenia. En los noventa, las economías en desarrollo se caracterizaron por el "miedo a flotar" mientras que, en la primera década de los 2000, predominaron el "miedo a apreciar" (Levy Yeyati y Sturzenegger, 2007: 2) y el "miedo a perder reservas" (Aizenman y Hutchison, 2010).

Como fue comentado, no es sencillo determinar la motivación principal en la demanda de reservas externas. Es probable que coexistan las diferentes motivaciones; sin embargo, a partir de la crisis asiática, los países en desarrollo han acumulado reservas preferentemente en carácter de autoseguro (Aizenman y Lee, 2005:3). En América Latina, la acumulación de reservas ha respondido preferentemente a motivos precautorios mientras que Argentina es un claro ejemplo de acumulación por motivos mercantilistas (Frenkel y Rapetti, 2010:41).

Varias son las opiniones respecto a los costos y beneficios de las políticas de intervención. Rodrik (2006:3) asegura que, en los países en desarrollo, tanto la acumulación de reservas como la reducción de las obligaciones externas de corto plazo permiten alcanzar liquidez y que, aun cuando estos países no han pagado un precio alto para protegerse frente a las crisis, una disminución aún mayor de sus obligaciones habría permitido alcanzar un mismo nivel de liquidez sorteando su alto costo económico y social. En general, se encuentra que las intervenciones son efectivas, aunque tal efectividad decrece en economías con altos niveles de inflación y de obligaciones externas dolarizadas (Daude, Levy Yeyati y Nanengast, 2014:18-19). 
La estrategia de acumulación de reservas es sumamente ventajosa, aunque también puede involucrar grandes costos. Dicha estrategia genera pérdidas cuasifiscales importantes en economías con elevadas prima riesgo-país y tasas de interés internas. Además, se halla sujeta a las ganancias y pérdidas de capital resultantes de las variaciones en los tipos de cambio entre las principales monedas de reserva (Ocampo, 2009:204). Sin embargo, como fue evidente en la reciente crisis financiera global, las economías en desarrollo se han favorecido con la instrumentación de esta política. En las economías de la región, la acumulación de reservas en moneda extranjera junto a la considerable reducción de los coeficientes de deuda externa en relación con el producto proporcionó un amplio margen de maniobra para la aplicación de políticas contra-cíclicas. La ruptura con el enfoque de total flexibilidad cambiaria y, especialmente, la determinación por no permitir que el tipo de cambio refleje totalmente el auge de los flujos de capital fue una pieza crucial en el manejo eficaz de dicha crisis (Ocampo, 2011:21-22).

En suma, el manejo de la política cambiaria por parte de los gobiernos de América Latina frente a turbulencias externas muestra un avance significativo vis-á-vis la experiencia histórica. Por cierto, la mayor flexibilidad de la política cambiaria constituye uno de los logros más significativos de la política macroeconómica de la región en los años 2000.

\section{Más allá del panorama local}

A la luz de lo expuesto, las economías en desarrollo están sujetas a grandes imperfecciones del sistema monetario internacional. Estas economías han buscado protegerse frente a los avatares de los mercados financieros internacionales por la vía de una agresiva política de acumulación de reservas en un nuevo enfoque de política cambiaria. A nivel global, con relación a la acumulación de reservas, los bancos centrales han estado interviniendo en los mercados de cambios a fin de evitar que los shocks comerciales y financieros causen fuertes fluctuaciones cambiarias. Tal acumulación se ha llevado a cabo en el marco de superávits en cuenta corriente - particularmente de China - por parte de los países conn relación a Estados Unidos, al tiempo que este país ha encontrado gran facilidad para financiar su déficit en cuenta corriente. Ciertamente, el apetito de los bancos extranjeros por títulos del Tesoro de dicho país ha sido una fuente inmediata de financiamiento externo de los déficits en cuenta corriente de los Estados Unidos, que crecieron desmesuradamente en los años anteriores a la explosión de la crisis global, lo que refleja el problema de desequilibrios globales (Eichengreen, 2009:54). Aunque tales desequilibrios han disminuido respecto a los niveles alcanzados en los años de la crisis global (FMI, 2019:135), su persistencia es aún fuente de preocupación.

En relación con la elección de los regímenes cambiarios, la diversidad de acuerdos cambiarios agrega complejidad al sistema monetario internacional. La alta movilidad de capitales torna inviable un sistema de tipos de cambios fijos a nivel global. Por su parte, frente a un sistema de flotación no administrada, uno de flotación administrada se muestra como más efectivo. Este podría adoptarse sobre la base de un acuerdo internacional sobre un conjunto de tipos de cambio que sirvieran como tipos de cambio de referencia. Ello redundaría en un mejor sistema monetario internacional y sería beneficioso tanto para los países avanzados como para los países emergentes y en desarrollo. La existencia de un tipo objetivo avalado internacionalmente - la tasa de referencia - facilitaría el funcionamiento del 
sistema. La mutua intervención puede ser más efectiva que la intervención unilateral, porque mostraría que los hacedores de política concuerdan respecto a la dirección a seguir. Del mismo modo, uno esperaría que una tasa de referencia acordada ayude a las autoridades a defender el objetivo anunciado. También permitiría a los países que han llegado a la conclusión de que la movilidad del capital se ha vuelto demasiado problemática tener un régimen que, aunque operase con márgenes difíciles, evitaría arrojarlos a los caprichos del mercado (Williamson, 2008:33).

La agenda de los foros globales ha avanzado considerablemente en varios temas. Tras la crisis global de 2008-2009, se verificó un importante avance en lo que atañe a la provisión de bienes públicos en el área financiera, lo que incluye la cooperación entre los principales centros financieros, un significativo movimiento hacia la armonización de regulaciones financieras entre los países, un papel mayor de las instituciones financieras internacionales y otras medidas asociadas con la estabilidad financiera a nivel doméstico (Frieden, 2017:533). Sin embargo, la provisión de bienes públicos en lo que atañe al área monetaria mostró un progreso menor. Una configuración compleja de la gobernanza internacional dificulta la optimización de los resultados en esta área. Aunque el escepticismo en materia de cooperación internacional es alto, también debe recordarse que la humanidad está condenada a la cooperación (Wolf, 2014).

\section{Conclusión}

El cambio del sistema monetario internacional posterior a los Acuerdos de Bretton Woods trajo aparejados beneficios, aunque también costos. Por un lado, en el marco de una alta movilidad de capital, el pasaje a un sistema de tipos de cambio flotantes se propuso superar la rigidez del sistema anterior y, con ello, permitió a los gobiernos llevar a cabo una política monetaria autónoma. Por otro lado, los problemas generados por los desequilibrios globales han sido una fuente importante de preocupación en diferentes períodos, lo que ha dado lugar a debates sobre la necesidad de mecanismos de ajuste apropiado con los tipos de cambio como foco de atención.

El sistema monetario internacional actual presenta varios problemas. Un primer problema se refiere a la persistencia de las asimetrías entre los países superavitarios y los deficitarios, así como entre los que son emisores de las monedas de reserva y los que no lo son. Un segundo problema consiste en la inestabilidad asociada al uso de una moneda nacional como moneda internacional, de modo que la economía mundial se halla sujeta a la política monetaria completamente autónoma del país emisor de la moneda de reserva dominante. El tercer problema es el carácter inequitativo del sistema, puesto que la gran acumulación de reservas por parte de los países en desarrollo se invierte en la adquisición de activos emitidos por los países ricos, lo que implica una transferencia de recursos hacia dichos países a tasas de interés bajas. Los países que no pueden tomar préstamos en su propia moneda encuentran dificultades para participar en el movimiento hacia una mayor flexibilidad del tipo de cambio. A su vez, el centro del sistema muestra una situación privilegiada en virtud de su percepción de señoreaje.

El cambio del sistema monetario internacional desde los tipos de cambio fijos a otro de tipos de cambio flotantes dista del proyecto ideado por los artífices del rediseño de la 
arquitectura financiera internacional. Los acuerdos cambiarios han proliferado y puede decirse que el sistema monetario internacional es una especie de "no-sistema" que ha experimentado crisis financieras y económicas recurrentes con los tipos de cambio como factores claves en la generación de tales eventos disruptivos. Frente a este contexto global, los países en desarrollo se han auto-asegurado por la vía de la acumulación de reservas en moneda extranjera. Sin embargo, esto ha sido uno de los aspectos más distintivos de los desequilibrios globales.

En su lucha antiinflacionaria, las economías de América Latina han aplicado sistemas cambiarios fijos y semifijos, pero las diversas crisis han mostrado las limitaciones que ellos presentan. Desde los 2000, las economías de la región se han movido hacia regímenes cambiarios más flexibles y varios anunciaron la implementación de metas de inflación. Sin embargo, la intervención cambiaria no está ausente. Actualmente, la política cambiaria en marcha es un activo importante para América Latina. La mayor flexibilidad de los tipos de cambio junto a la persistencia en la acumulación de reservas externas permitió a las economías de la región amortiguar los shocks externos e internos de manera eficaz, lo que difiere significativamente de lo acontecido en situaciones críticas del pasado.

Más allá del ámbito local, temas cruciales del sistema monetario internacional, como los desequilibrios globales o la política cambiaria global, están aún en la agenda de los foros globales. Puesto que la arquitectura financiera global ha estado exhibiendo grandes imperfecciones, es oportuno reflexionar sobre la necesidad de afianzar el consenso y la cooperación internacional a fin de conferir mayor coherencia a dicho sistema y de este modo optimizar los resultados globales.

\section{Bibliografía}

Aizenman, J. y J. Lee (2005), "International Reserves: Precautionary versus Mercantilist Views, Theory and Evidence", documento de trabajo n¹1366, Cambridge, MA: National Bureau of Economic Research.

Aizenman, J. y M. M. Hutchison (2010), “Exchange Market Pressure and Absorption by International Reserves: Emerging Markets and Fear of Reserve Loss during the 2008-09 Crisis", documento de trabajo no 16260, Cambridge, MA: National Burau of Economic Research.

Alberola, E. y J. M. Serena (2010), “Evolución y perspectivas sobre los desequilibrios globales en el nuevo escenario internacional", Revista de Economía, Madrid, no 855; pp. 27-41.

Calvo, A. G. y C. M. Reinhart (1999), "Fear of Floating”, documento de trabajo n7993, Cambridge, MA: National Bureau of Economic Research.

Chinn, M. D. y S. Wei, “A Faith-Based Initiative: Does a Flexible Exchange Rate Regime Really Facilitate Current Account Adjustment? documento de trabajo n14420, Cambridge: MA: National Bureau of Economic Research.

Daude, C., E. Levy Yeyati y A. Nagengast (2014), “On the effectiveness of exchange rate interventions in emerging markets", documento de trabajo no 288, Cambridge, MA: Center for International Development. 
De la Torre, A., E. Levy Yeyati y S. Pienknagura (2013), Latin America's Deceleration and the Exchange Rate Buffer, Washington, DC: Banco Mundial.

Eichengreen, B., R. Hausmann y U. Panizza (2002), “Original Sin: The Pain, the Mistery, and the Road to Redemption", documento presentado en la Conferencia Currency and Maturity Matchmaking: Redeeming Debt from Original Sin, Washington, DC: Banco Interamericano de Desarrollo. http://www.financialpolicy.org/financedev/hausmann2002.pdf

Eichengreen, B. (2008), Globalizing Capital: A History of the International Monetary System, Princeton, NJ: Princeton University Press.

Eichengreen, B. (2009), "The Dollar Dilemma”, Foreign Affairs, New York, v.88; no5; pp. 52-68.

Eichengreen, B. (2011), Exorbitant Privilege: The Rise and Fall of the Dollar and the Future of the International Monetary System, New York: Oxford University Press.

Feldstein, M. (1999), "Self-Protection for Emerging Markets Economies", documento de trabajo no 6907, Cambridge, MA: National Bureau of Economic Research.

French Davis, R. (2003), "Políticas económicas, desarrollo nacional y crisis financieras", $R e$ vista de la Facultad de Ciencias Económicas de la UNMSM, Lima v. VIII; no 22; pp. 7-32.

Fischer, S. (2001), "Exchange Rate Regimes: Is the Bipolar View Correct?", Journal of Economic Perspectives, Pittsburgh, PA, v.15; no 2; pp. 1-24.

Fondo Monetario Internacional (2019), Perspectivas de la Economía Mundial, Washington, DC: Fondo Monetario Internacional.

Frankel, J. A. (2012), “Choosing an Exchange Rate Regime”, en James, J., I. W. Marsh y L. Sarno (eds.), Handbook of Exchange Rates, Nueva Jersey: Wiley Online Library, https://www.hks.harvard.edu/fs/jfrankel/ExRRegimes-MarshSarnoHdbkCh28.pdf

Frenkel, R. (1989), El régimen de alta inflación y el nivel de actividad, Buenos Aires: CEDES.

Frenkel, R. y M. Rapetti (2010), A Concise History of Exchange Rate Regimes in Latin America, Center for Economic and Policy Research (CEPR).

Frieden, J. A. (2017), "The Governance of International Finance", en Frieden, J. A., D. A. Lake y J. L. Broz (ed.), International of Political Economy, New York: W.W. Norton \& Company, pp. 518-534.

Ghosh, A. R., J. D. Ostry y M. S. Qureshi (2014), "Exchange Rate Management and Crisis Susceptibility: A Reassessment", documento de trabajo n14/11, Washington, DC: Fondo Monetario Internacional.

Ghosh, A. R., M. S. Qureshi y C. G. Tsangarides (2014), “Friedman Redux: External Adjustment and Exchange Rate Flexibility", documento de trabajo nำ14/146, Washington, DC: Fondo Monetario Internacional.

Hamada, K. (1985), The Political Economy of international monetary interdependence, Cambridge, MA: MIT Press.

Hausmann, R., M. Gavin, C. Pages Serra y E. Stein (1999), "Financial Turmoil and the Choice of 
Exchange Rate Regime”, CID documento de trabajo n400, Washington, DC: Banco Inter-Americano de Desarrollo.

Ilzetzki, E., C. M. Reinhart y K. S. Rogoff (2019), “Exchange Arrangements Entering the Twenty First Century: Which Anchor Will Hold?, Quarterly Journal of Economics, vol. 134 , no 2, pp. 599-646.

Johnson, H. G. (1958), International Trade and Economic Growth: Studies in Pure Theory, Londres: G. Allen \& Unwin.

Katz, S. (2009), "Centro y Periferia: La propagación de la crisis a las economías latinoamericanas", Ensayos Económicos, Buenos Aires, no 53-54; pp. 117-166.

Krugman, P. (1979), “A Model of Balance Payment Crisis”, Journal of Money, Credit and Banking, v.11; n우; pp. 311-325.

Krugman, P. (2000), “Currency Crises”, en P. Krugman (ed.), Currency Crises, Chicago: University of Chicago Press.

Krugman, P. R., M. Obstfeld y M. J. Melitz (2012), Economía Internacional: teoría y política, Madrid: Pearson Educación.

Levy Yeyati, E. (2011), “Exchange Rate Regimes”, CIF documento de trabajo no02/2011, Buenos Aires: Universidad Torcuato Di Tella.

Levy Yeyati, E. y F. Sturzenegger, (2007), "Fear of Appreciation", documento de trabajo no 4387, Washington, DC: Banco Mundial.

Li, J. y R. S. Rajan (2005), "Can High Reserves Offset Weak Fundamentals? A Simple Model of Precautionary Demand for Reserves", documento de trabajo n509, Adelaida: Centre for International Economic Studies.

Machinea, J. L. y J. M. Fanelli (1988), “El control de la hiperinflación”, en Bruno, M., G. Di TeIla, R. Dornbusch y S. Fischer (eds.), Inflación y Estabilización La Experiencia de Argentina, Bolivia, Israel y México, México: Fondo de Cultura Económica, pp. 141-188.

Martin, L. L. y B. A. Simmons (1999), "Theories and Empirical Studies of International Institutions", en Katzenstein, P.J., R.O. Kehoane y S.D. Kramer (eds)., Exploration and Contestations in the Study of World Politics, Cambridge, MA: MIT Press, pp. 729-757.

Matoo A. y A. Subramanian (2008), "Currency Undervaluation and Sovereign Wealth Funds", documento de trabajo no 08-2, Washington, DC: Peterson Institute for International Economics

Naciones Unidas (2001), "Report of the High-Level Panel on Financing for Development" (Zedillo Report), New York: United Nations, recuperado de http://www.un.org/en/events/pastevents/financing_for_development_report.shtml

Obstfeld, M. y K. Rogoff (1995), "The Mirage of Fixed Exchange Rates", documento de trabajo no 5191, Cambridge, MA: National Bureau of Economic Research. 
Ocampo, J. A. (2002), Developing Countries' Anticyclical Policies in a Globalized World, Santiago de Chile: Naciones Unidas- CEPAL.

Ocampo, J. A. (2009), “Implicaciones de política económica de la inestabilidad financiera actual”, Ensayos Económicos, Buenos Aires, no53-54; pp. 191-215.

Ocampo, J. A. (2011), “¿Cómo fue el desempeño de América Latina durante la crisis financiera global?”, Ensayos Económicos, Buenos Aires, no61-62; pp. 7-33.

Ocampo, J. A. (2016), "A brief history of the international monetary system since Bretton Woods", documento de trabajo no 97, Helsinki: UNU-WIDER.

Ocampo, J. A. (2017), Resetting the International Monetary (non) System, Oxford: Oxford University Press.

Olivera, J. G.H. (1969), "A Note on the Optimal Rate of Growth of International Reserves", Journal of Political Economy, v.77; no2; pp. 245-248.

Perry, G. y L. Servén (2003), The Anatomy of a Multiple Crisis: Why Was Special and What Can We Learn from it?, Washington, DC: Banco Mundial.

Ramos, J. (1985), "Stabilization and Adjustment Policies", CEPAL Review, Santiago de Chile no25; pp. 85-108.

Rodrik, D. (2006), "The Social Cost of Foreign Exchange Reserves", documento de trabajo no 11952, Cambridge, MA: National Bureau of Economic Research.

Rodrik, D. (2008), "The Real Exchange Rate and Economic Growth", Brookings Papers on Economic Activity, Washington, DC, no2; pp. 365-412.

Rojas-Suárez, L. (2010), "The International Financial Crisis: Eight Lessons for and from Latin America", documento de trabajo n202, Washington, D.C.: Center for Global Development.

Salvatore, D. (2011), International Economics Trade and Finance, Singapore: John Wiley \& Sons.

Stein, A. A. (1990), Why Nations Cooperate: Circumstance and Choice in International Relations, Ithaca: Cornell University Press.

Triffin, R. (1947), "National Central Banking and the International Economy", Postwar Economic Studies, St. Louis, nำ, pp. 46-81.

Triffin, R. (1968), El Sistema monetario internacional, Buenos Aires: Amorrortu.

Williamson, J. H. (1977), The failure of world monetary reform 1971-1974, Nairobi: NG Nelson.

Williamson, J. (2008), "Exchange Rate Economics", documento de trabajo № 08-3, Washington, DC: Peterson Institute for International Economics, Institute of International Economics.

Wolf, M. (2014), "Three events that shaped our world", Financial Times, Londres, 10 de junio. 